Genetic Improvement of Bioenergy Crops 
Wilfred Vermerris

Editor

\section{Genetic Improvement of Bioenergy Crops}

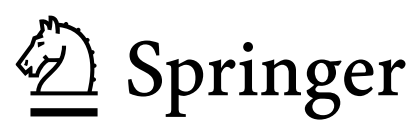




\section{Editor}

Wilfred Vermerris

University of Florida

Gainesville, FL

USA

ISBN: 978-0-387-70804-1

e-ISBN: 978-0-387-70805-8

DOI: $10.1007 / 978-0-387-70805-8$

Library of Congress Control Number: 2008930840

(C) 2008 Springer Science+Business Media, LLC

All rights reserved. This work may not be translated or copied in whole or in part without the written permission of the publisher (Springer Science+Business Media, LLC, 233 Spring Street, New York, NY 10013, USA), except for brief excerpts in connection with reviews or scholarly analysis. Use in connection with any form of information storage and retrieval, electronic adaptation, computer software, or by similar or dissimilar methodology now known or hereafter developed is forbidden.

The use in this publication of trade names, trademarks, service marks, and similar terms, even if they are not identified as such, is not to be taken as an expression of opinion as to whether or not they are subject to proprietary rights.

Cover illustration: The poplar image was provided by Dr. Brian Stanton (GreenWood Resources) and the sorghum image was provided by Dr. Ana Saballos (University of Florida).

Printed on acid-free paper

springer.com 


\section{Preface}

Bioenergy is receiving increasing attention in science, politics, industry and the media. As a result, bioenergy is no longer an obscure topic pursued by ideologists striving for a green economy in which everyday luxuries have to be sacrificed. Instead, the bioeconomy is touted as a new economic model that offers green growth, i.e. economic opportunities that rely on sustainable, environmentally-friendly production and consumption.

Several factors have contributed to this change. Global climate change, once a hotly debated topic, appears to have become accepted as a real phenomenon with potentially catastrophic consequences for both the environment and the global economy. The change in perception of global climate change is the result of intense research and effective communication of that research by many people, including the 2007 Nobel Peace Prize laureates, the United Nations International Panel on Climate Change (IPCC) and former United States vice-president Al Gore. Other developments that have contributed to this awareness include the devastating impact of hurricane Katrina in 2005, massive forest fires in the western and southeastern United States due to prolonged drought, and the realization that weather patterns in many parts of the world in the recent years seem more erratic. In addition, crude oil prices, which have been stable for decades between US\$25 and \$35 per barrel, are now closer to US $\$ 80$ per barrel, and on January 4, 2008 hit the much-dreaded price of US\$100 per barrel. This increase in the oil price is indicative of the fact that oil reserves are finite, while global demand for oil will continue to rise. Given that oil is used on a global scale but that oil production is controlled by a limited number of countries, individual countries have very little influence of oil prices.

This book was written with the belief that energy production and use based on the 'business-as-usual' model are not sustainable in the future, due to the impact on the climate, the growing world population, and the fact that oil reserves are finite. Bioenergy is not a magic solution to the world's energy needs, but it has the potential to contribute significantly to these needs, when used in combination with conservation programs and other sources of alternative energy. 
In order for bioenergy to become a reality, a lot of elements need to be put in place. This includes the infrastructure to harvest, transport and process biological materials, the infrastructure to distribute the fuels and green chemical feedstocks produced from these materials, and the vehicles and down-stream processes that use the bioenergy. At the base of this bioenergy production chain are the plant feedstocks. At the time of this writing, the two main sources of bioenergy are corn starch in the United States and sugar from sugar cane in Brazil. Given the anticipated increase in the demand for bioenergy, and the concern that food and feed security are at risk if corn starch and sugar continue to be the main feedstocks, the consensus is that lignocellulosic biomass is a better feedstock for the long term. At this time, the technology to produce fuels from these feedstocks is not mature, and the feedstocks themselves have not been developed for bioenergy production per se. Bioenergy production can therefore become much more efficient if both the feedstocks and the processing technology are optimized. The main focus of this book is on the genetic strategies that are available for improving bioenergy crops that can be used for the production of ethanol and other chemical derived from lignocellulosic biomass.

This book is divided into two parts. Part I provides background information on bioenergy production and includes a description of the current practice for ethanol production from grain, what the processing options are when lignocellulosic biomass is used, what lignocellulosic biomass is and how it is synthesized in the plant, and how you can chemically characterize and evaluate biomass. Part I also contains a primer on plant breeding and genetics for those readers that are unfamiliar with these topics. Part II provides an overview of a number of important bioenergy crops. Each chapter covers one crop or a group of related crops, and describes the biology, crop use and crop potential, genetic strategies and plant breeding efforts as they relate to bioenergy production. The focus of this book is on so-called biomass crops, where carbohydrates provide the main source of energy. This can be either in the form of sugar used directly in microbial fermentation or in form of cell wall polysaccharides that are processed to fermentable sugars or that are used in thermo-chemical processing (syngas, co-firing). Oil crops for the production of bio-diesel are not covered in this book, largely because the chemistry and processing are very different, and this would likely have resulted in a lack of focus. While bioenergy is of global interest, many of the chapters have a focus on production in the United States. This is the result of several recent initiatives in this country that have led to a stimulus of research on genetic improvement and bioprocessing and that have also resulted in the construction of many new processing plants. The selection of crops represented in Part II is based on the potential for large-scale bioenergy production, and on the presence of active crop improvement programs with a focus on bioenergy. Obviously, some crops not represented in this book may become suitable bioenergy crops in the future, especially after several years of research and breeding.

The book is designed for use as a textbook for a course on bioenergy production at the graduate student level. At the University of Florida this book is used for a course with the same name as the book, offered through the department of Agronomy, and open to students from other departments in the plant sciences, as well as students in chemical and biological engineering with an interest in learning 
more about feedstock composition and modification. This three-credit course covers more or less one chapter per week, and is supplemented with articles from the primary literature. While this book is written as a text book, it will also be of interest for researchers at academic institutions, government research facilities, and private industry as well as for policy makers in the bioenergy area.

As the editor of this book, I would like to thank the chapter authors for their valuable contributions, Randi Wheeler for her assistance with the editing and reference formatting, Miguel Castillo and Nesbit Tyler for proofreading chapters, and Jinnie Kim and Jillian Slaight at Springer for their encouragement and flexibility. This book would not have been possible without the support at home from Lauren and Deirdre. I am grateful for their patience, especially when the writing took place on evenings and in weekends. This book is dedicated to my (currently) 7-year old daughter Deirdre, as a representative of the next generation that will have to face the consequences of the energy-related and environmental choices the current generation makes.

Wilfred Vermerris

Gainesville, FL, USA

Spring 2008 


\section{Contents}

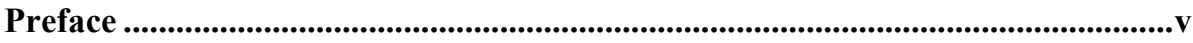

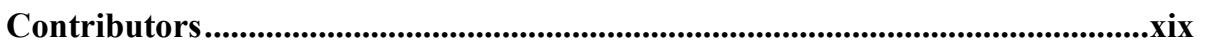

\section{Part I}

1. Why Bioenergy Makes Sense ........................................................................................3 Wilfred Vermerris

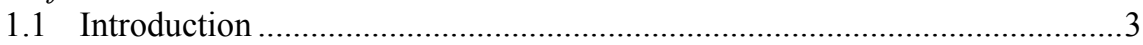

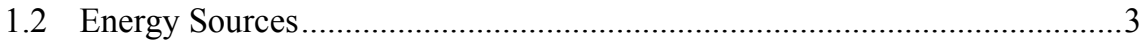

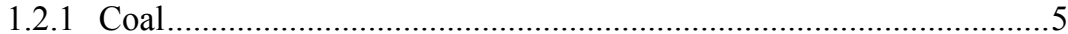

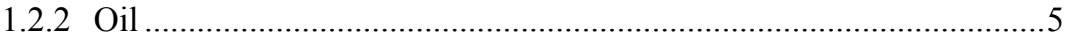

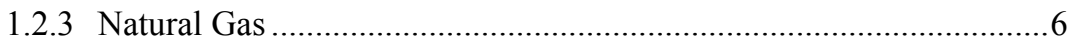

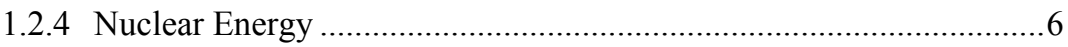

1.2.5 Hydroelectric Energy .....................................................................

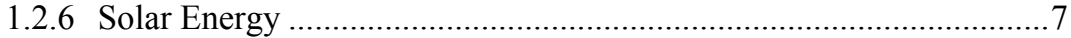

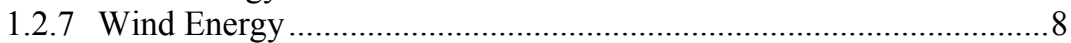

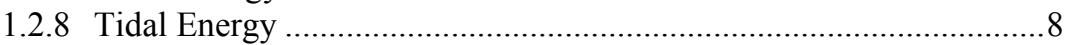

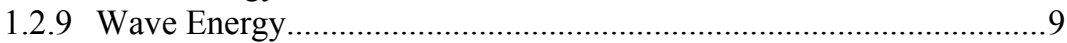

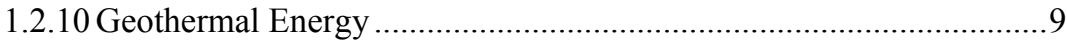

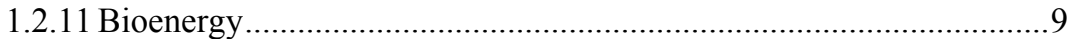

1.3 Photosynthesis: Capturing Solar Energy in Chemical Bonds .......................12

1.3.1 $\mathrm{C}_{3}$ Photosynthesis .....................................................................12

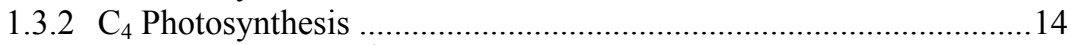

1.3.2.1 The NADP ${ }^{+}$-Malic Enzyme Variant ......................................15

1.3.2.2 The NAD ${ }^{+}$-Malic Enzyme Variant.........................................16

1.3.2.3 The Phosphoenolpyruvate Carboxykinase Variant ...............17

1.3.2.4 Biosynthesis of Biofuel Feedstocks from

Photosynthate 
1.4 Alternative Energy to Meet Future Global Energy Needs..........................19

1.4.1 Reducing Global Carbon Emissions ..............................................19

1.4.2 Political and Economic Motivations for Using Alternative Energy ..................................................................................24

1.5 Initiatives Around the World to Stimulate Bioenergy Production................26

1.6 Bioenergy, Oil Companies, and Car Manufacturers ...................................29

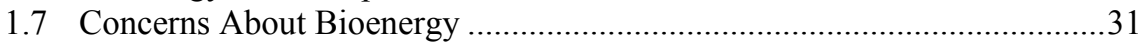

1.7.1 Food Versus Fuel ........................................................................ 31

1.7.2 Negative Net Energy Balance ................................................... 32

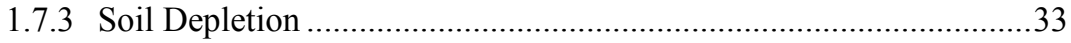

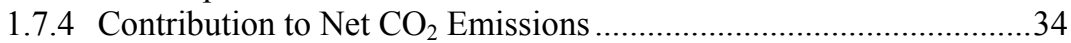

1.7.5 Biofuels Prolong the Power of the Oil Companies ...........................35

1.7.6 Ethanol is an Uneconomical Fuel ..................................................36

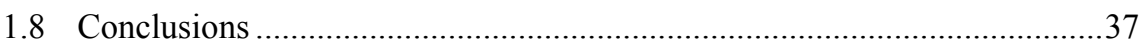

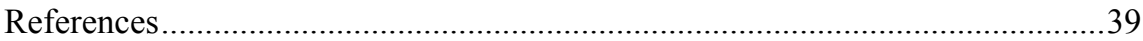

2. A Primer on Genetics, Genomics and Plant Breeding ..................................43 Wilfred Vermerris

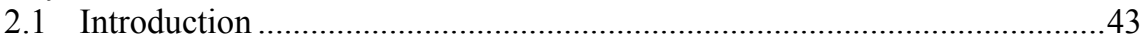

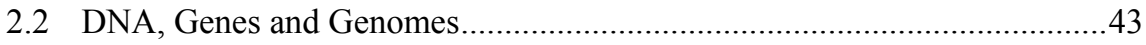

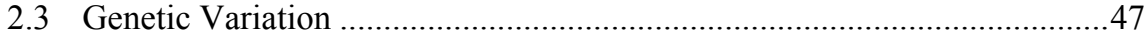

2.4 Molecular Markers ...................................................................................49

2.4.1 Definition and Use ...................................................................49

2.4.2 The Polymerase Chain Reaction .....................................................49

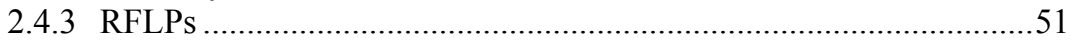

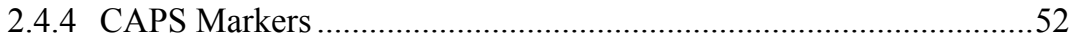

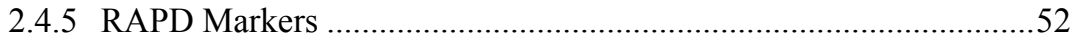

2.4.6 SSR or Microsatellite Markers......................................................5

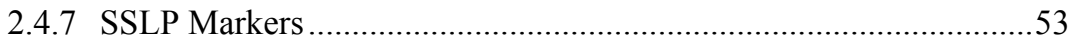

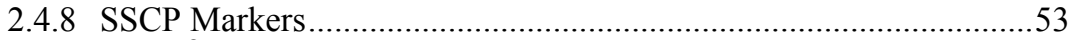

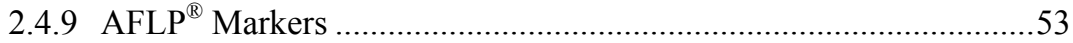

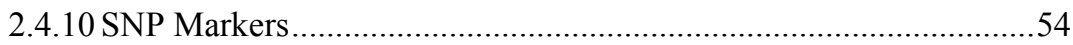

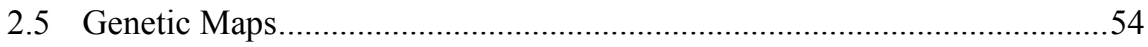

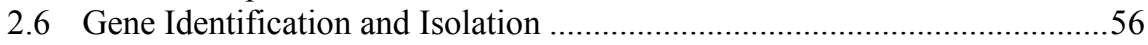

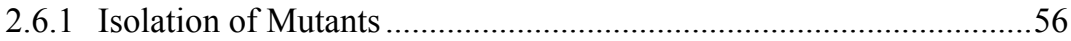

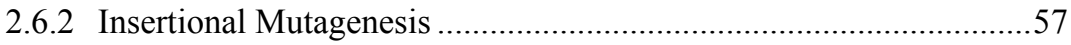

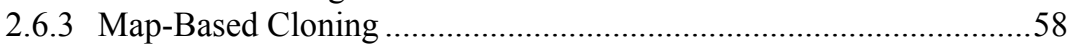

2.6.4 The Candidate-Gene Approach.....................................................59

2.6.5 Gene Identification Based on Differential Gene Expression .............59

2.6.5.1 Differential Display .................................................60

2.6.5.2 Subtractive Hybridization ...............................................60

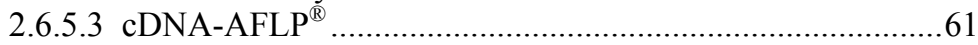

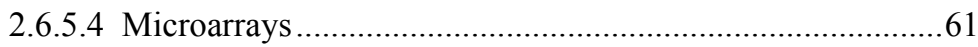

2.6.5.5 High-Throughput cDNA Sequencing .............................61

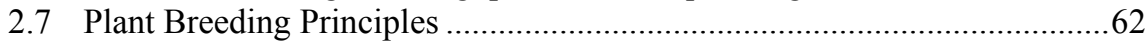

2.7.1 Identification and Combination of Genetic Variation.......................62

2.7.2 Quantitative Traits ..................................................................63 
2.7.3 Selection 64

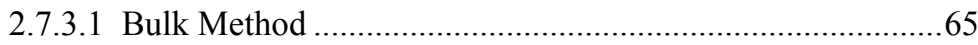

2.7.3.2 Single-Seed Descent Method ..............................................65

2.7.3.3 Mass Selection ....................................................................66

2.7.3.4 Pedigree Method .................................................................66

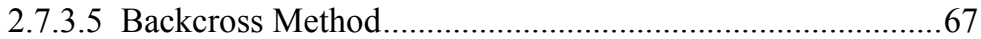

2.7.4 Testing and Evaluation .................................................................6 68

2.7.5 Cultivar Production....................................................................6 69

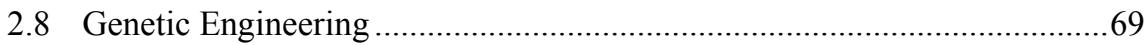

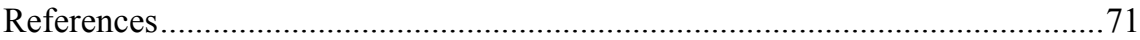

3. Production of Ethanol from Grain ..................................................................75

Nancy N. Nichols and Rodney J. Bothast

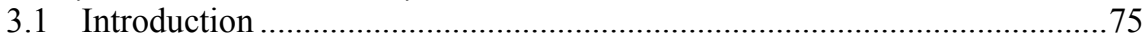

3.2 Ethanol Fermentation Processes ................................................................ 75

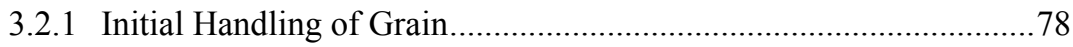

3.2.2 Conversion of Starch to Fermentable Sugars.................................78

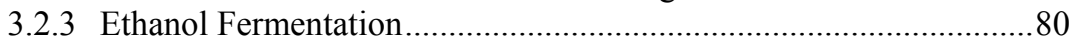

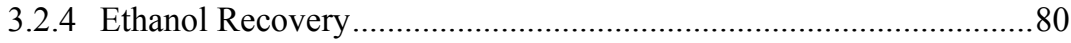

3.2.5 Stillage Processing ...................................................................... 81

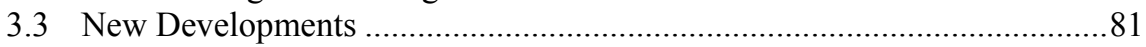

3.3.1 Milling and Separation Technologies ..........................................81

3.3.2 New Enzymes and Yeast Strains ................................................. 82

3.3.3 New Corn Hybrids ..........................................................................83

3.3.4 Co-product Quality and Utilization...............................................83

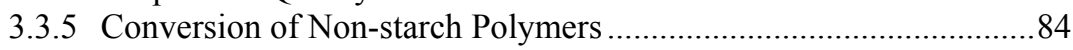

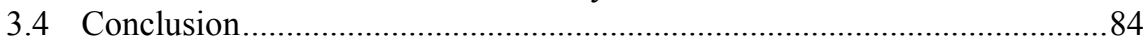

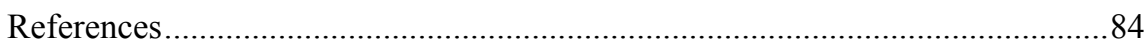

4. Composition and Biosynthesis of Lignocellulosic Biomass...........................89

Wilfred Vermerris

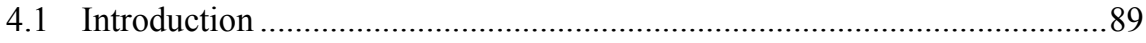

4.2 Lignocellulosci Biomass is Composed of Plant Cell Walls........................89

4.3 Carbohydrate Nomenclature ......................................................................91

4.3.1 The +/- -Nomenclature ............................................................ 91

4.3.2 The L/D -Nomenclature .......................................................... 92

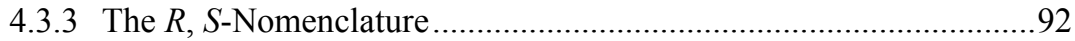

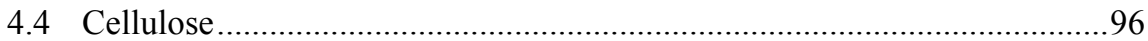

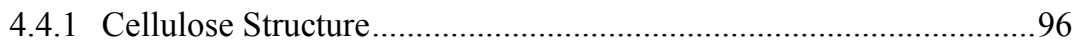

4.4.2 Cellulose Biosynthesis ............................................................98

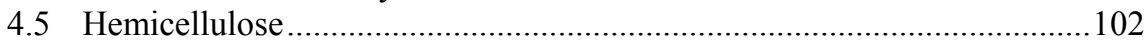

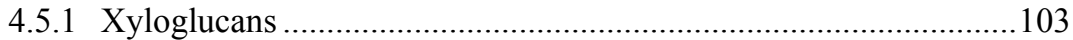

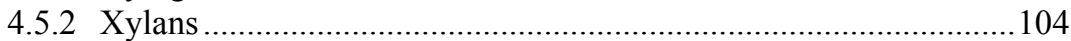

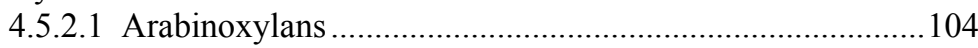

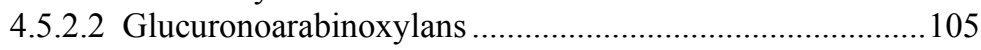

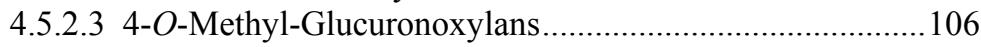




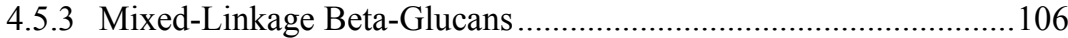

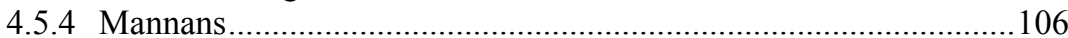

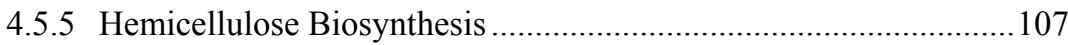

4.5.5.1 The Nucleotide Sugar Interconversion Pathway ................108

4.5.5.2 Biosynthesis of Hemicellulosic Polysaccharides ...............111

4.5.5.3 Xyloglucan Modification ...................................................... 115

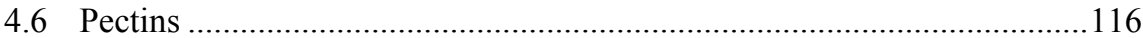

4.7 Lignin and Hydroxycinnamic Acids................................................... 117

4.7.1 Biosynthesis of Monolignols and Hydroxycinnamic Acids............117

4.7.2 Monolignol Transport ............................................................. 120

4.7.3 Monolignol Oxidation and Polymerization.................................... 121

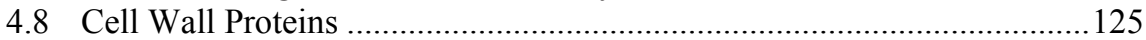

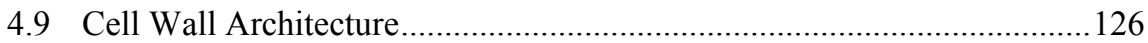

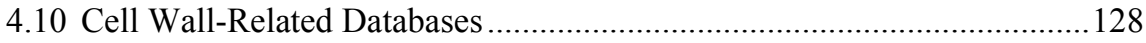

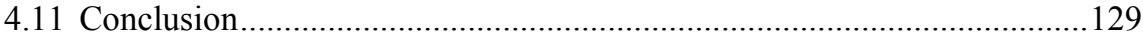

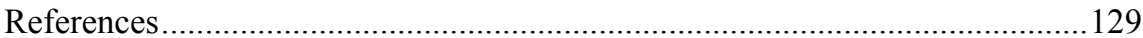

5. Selection of Promising Biomass Feedstock Lines Using High-

Throughput Spectrometric and Enzymatic Assays........................................143

Mark F. Davis, Ed Wolfrum and Tina Jeoh

5.1 Introduction ....................................................................................... 143

5.2 Integrating Spectroscopy and Multivariate Statistical Data Analysis

for High-Throughput Cell Wall Chemical Analysis ................................ 143

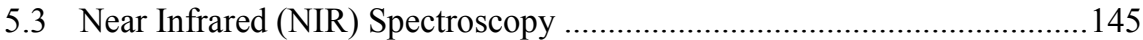

5.3.1 Description of Instrumentation ................................................... 145

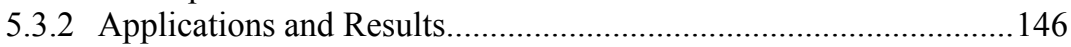

5.3.2.1 Qualitative Data Analysis (Classification) .......................146

5.3.2.2 Quantitative Data Analysis (Prediction) ...........................147

5.4 Pyrolysis-Molecular Beam-Mass Spectrometry .................................... 148

5.4.1 Description of Instrumentation ................................................... 148

5.4.2 Applications and Results.......................................................... 150

5.4.2.1 Estimates of Lignin Composition and Structure ...............150

5.4.2.2 Detection of Quantitative Trait Loci .............................. 152

5.4.2.3 Chemical Compositional Changes after Pretreatment and Biological Conversion ............................................. 152

5.4.2.4 Screening for Unintended Effects .................................. 154

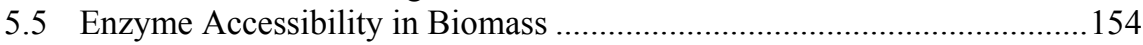

5.5.1 Probing for Enzyme Accessibility in Biomass............................ 154

5.5.2 Applications and Results......................................................... 155

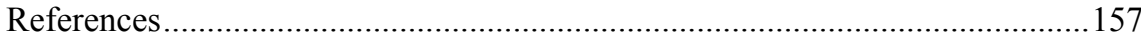

\section{Current Technologies for Fuel Ethanol Production from}

Lignocellulosic Plant Biomass

Yulin Lu and Nathan S. Mosier

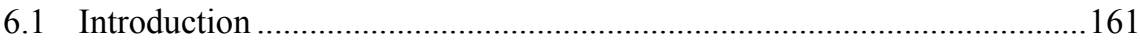

6.2 Feedstock Pretreatment Strategies ................................................... 163 
6.2.1 Neutral/Controlled-pH Pretreatment............................................ 165

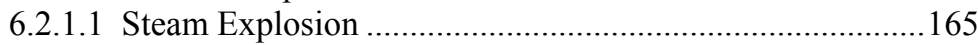

6.2.1.2 Liquid Hot Water Pretreatment ........................................ 165

6.2.1.3 Controlled-pH Pretreatment .............................................. 166

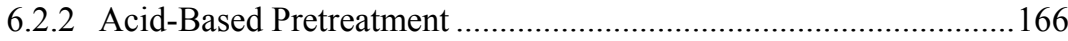

6.2.2.1 Dilute Acid Pretreatment .................................................... 166

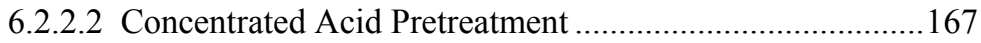

6.2.3 Alkaline-Based and Other Pretreatments .................................... 167

6.2.3.1 Lime ............................................................................ 167

6.2.3.2 Ammonia Fiber Expansion ............................................. 168

6.3 Enzymatic Hydrolysis: Liberating Monosaccharides ................................168

6.4 Ethanol Fermentation: Strain Development for Sugar

Co-fermentation................................................................................. 171

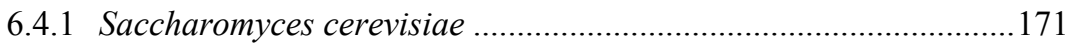

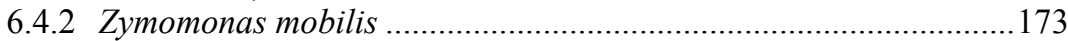

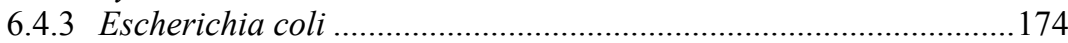

6.5 Ethanol Recovery: Distillation and Dehydration..................................... 175

6.6 Perspectives on Advanced Biochemical Conversion Technologies ...........176

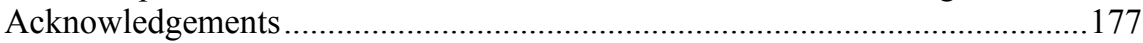

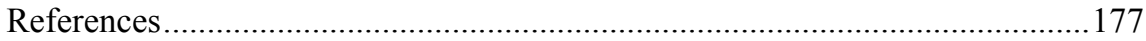

\section{Part II}

7. Genetic Improvement of Corn for Lignocellulosic Feedstock

Production

Natalia de Leon and James G. Coors

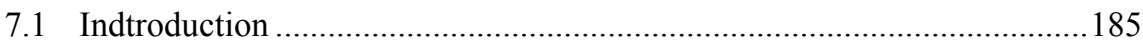

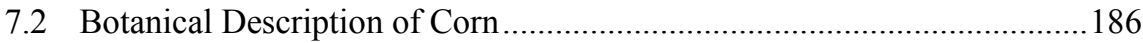

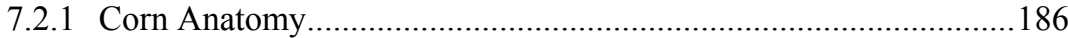

7.2.2 Corn Origin and Habiat................................................................ 187

7.2.3 Corn Reproduction and Biology .................................................. 188

7.2.4 Genome Structure and Organization...........................................190

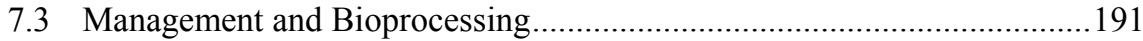

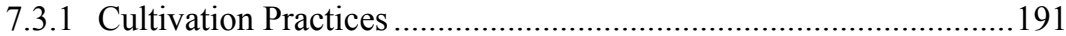

7.3.2 Biomass Yield Potential................................................................. 193

7.3.3 Biomass Processing ..................................................................... 195

7.4 Utilization of Corn Stover as a Source of Biomass ................................196

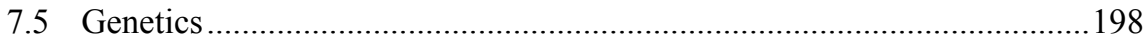

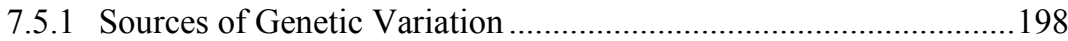

7.5.2 Use of Genetic Variation ...............................................................199

7.6 Current Research Efforts and Future Outlook ..........................................201

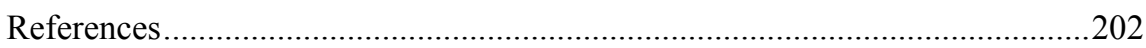

8. Development and Utilization of Sorghum as a Bioenergy Crop ..................211 Ana Saballos

8.1 Introduction ..................................................................................2 211

8.2 Botanical Description of Sorghum ..................................................212 


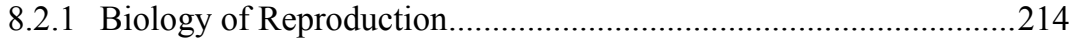

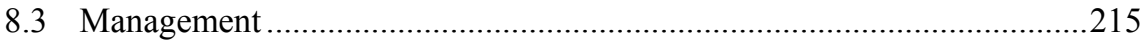

8.3.1 Agronomic Considerations ....................................................215

8.3.2 Pests and Diseases ..................................................................217

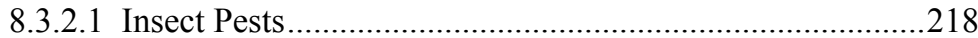

8.3.2.2 Diseases ......................................................................220

8.3.3 Harvest and Processing Systems ...............................................222

8.4 Current Status and Future Prospects....................................................223

8.5 Genetic Improvement of Sorghum ......................................................225

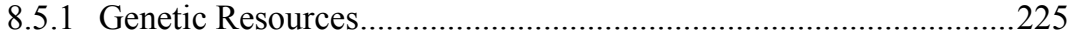

8.5.1.1 Natural Variation ...........................................................225

8.5.1.2 Mutants .....................................................................22 226

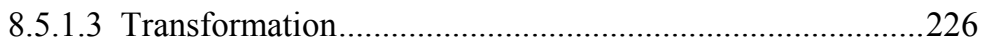

8.5.1.4 Sorghum Genomics.......................................................227

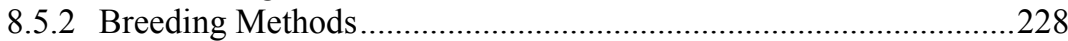

8.5.3 Traits of Interest for Improvement ..............................................230

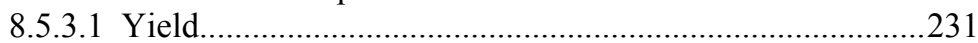

8.5.3.2 Stress Resistance...........................................................234

8.5.3.3 Moisture Content and Juice Extraction Efficiency............236

8.5.3.4 Conversion Efficiency of the Starch ..............................236

8.5.3.5 Cell Wall Composition..................................................236

8.5.3.6 Sugar Concentration.......................................................237

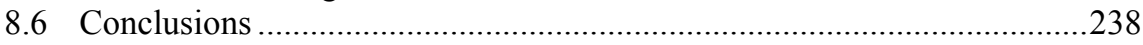

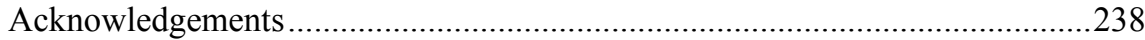

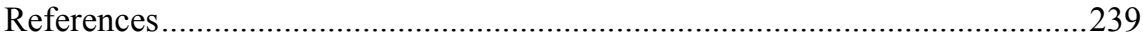

9. Genetic Improvement of Sugarcane (Saccharum spp.)

as an Energy Crop................................................................................................249

Thomas L. Tew and Robert M. Cobill

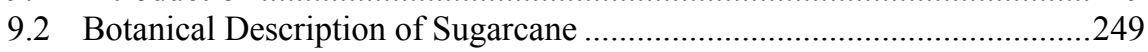

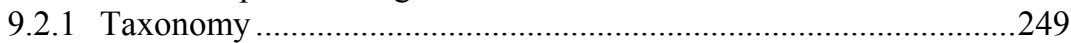

9.2.2 Sugarcane Cultivation and Harvest..............................................251

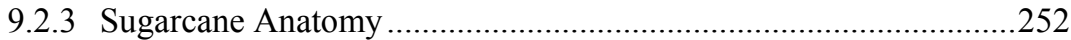

9.2.4 Early Breeding Efforts ............................................................254

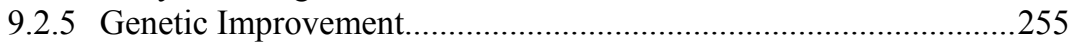

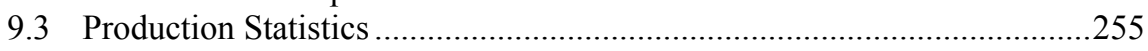

9.3.1 Worldwide Production Statistics.............................................25

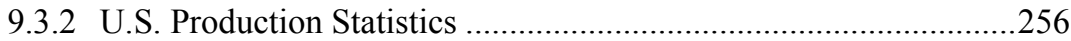

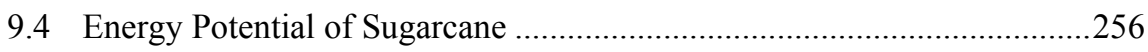

9.4.1 Yield Potential ..........................................................................256

9.4.2 Actual Yields .............................................................................257

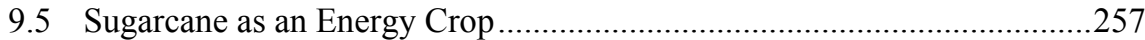

9.5.1 Sugar as a Feedstock ...............................................................25

9.5.2 Sugarcane Fiber as a Feedstock .................................................2. 258

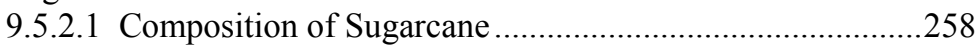


9.5.2.2 Boiler Fuel and Cogeneration .....................................259

9.5.2.3 Cellulosic Ethanol and Gasification..................................260

9.5.3 Energy Output/Input Ratio........................................................261

9.6 Energy Cane Breeding Strategies ........................................................262

9.6.1 The Sugar Model (Status Quo) .................................................262

9.6.1.1 Breeding for Sugar Yield and Improved Sugar

Content...................................................................2.262

9.6.1.2 Sugarcane Breeding in Brazil............................................263

9.6.2 Sugar and Fiber Model............................................................264

9.6.2.1 Type I Energy Cane Definition .........................................264

9.6.2.2 Genetic Base Broadening..................................................264

9.6.2.3 Caribbean and U.S. Experience ......................................265

9.6.3 Fiber-only Model (Type II Energy Cane) .....................................266

9.6.3.1 Type II Energy Cane Definition.........................................266

9.6.3.2 Breeding Within Saccharum Strictly for Fiber

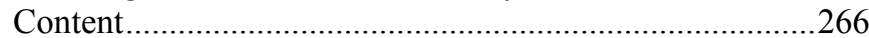

9.6.3.3 Related Genera and Intergeneric Hybridization.................267

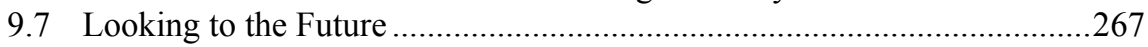

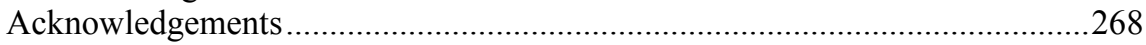

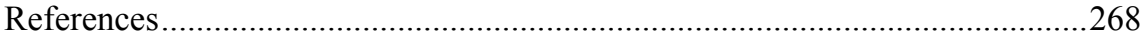

\section{Miscanthus: Genetic Resources and Breeding Potential to Enhance}

Bioenergy Production ....................................................................................273

John Clifton-Brown, Yu-Chung Chiang and Trevor R. Hodkinson

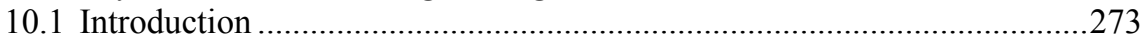

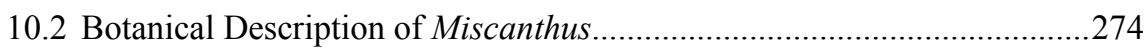

10.3 Agronomic Characteristics and Bioprocessing.....................................279

10.4 Production and Utilization...................................................................28

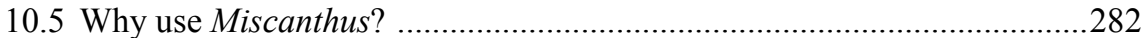

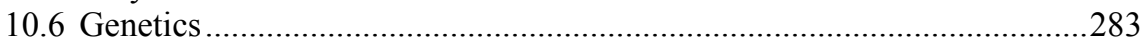

10.6.1 Using Genetic Variation for Breeding .........................................28

10.6.2 Traits of Interest...................................................................228

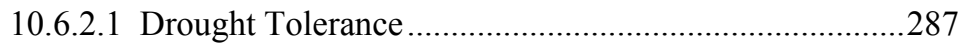

10.6.2.2 Frost Tolerance and Low Temperature Growth.............288

10.6.2.3 Flowering Time ......................................................28

10.6.2.4 Composition ..........................................................28

10.6.2.5 Propagation.............................................................2. 289

10.6.2.6 Pests and Disease .........................................................289

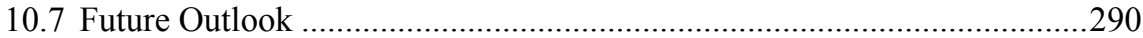

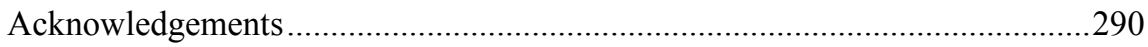

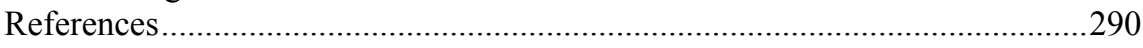

11. Improvement of Switchgrass as a Bioenergy Crop .....................................295

Joe Bouton

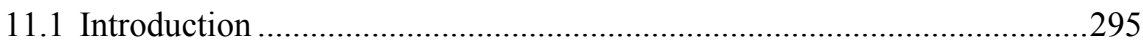

11.2 Rationale for using Switchgrass as a Bioenergy Crop..............................296 
11.3 Botanical Description of Switchgrass....................................................296

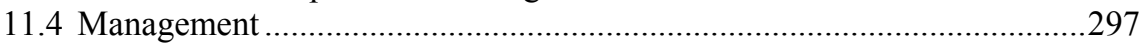

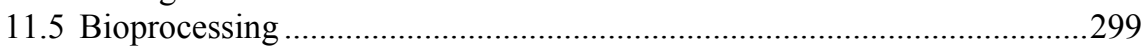

11.6 Breeding and Cultivar Development .....................................................300

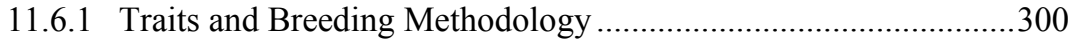

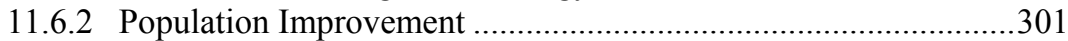

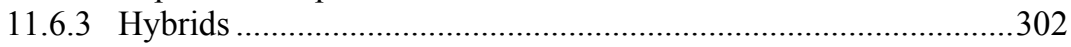

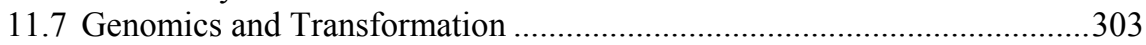

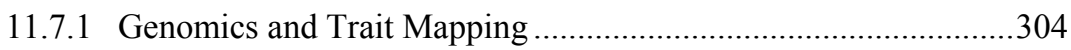

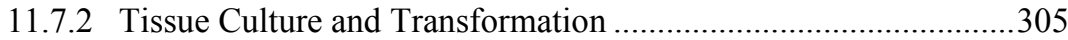

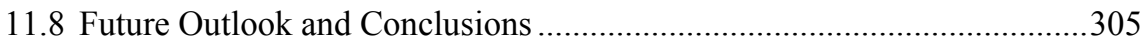

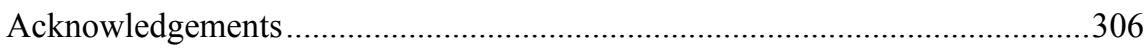

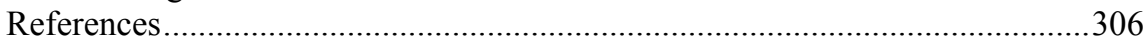

\section{Improvement of Perennial Forage Species as Feedstock}

for Bioenergy .........................................................................................................309

William F. Anderson, Michael D. Casler and Brian S. Baldwin

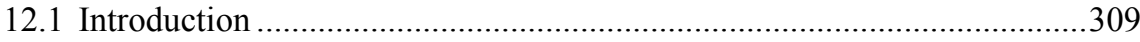

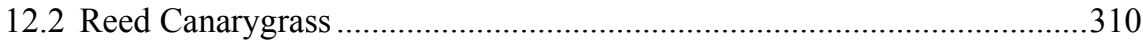

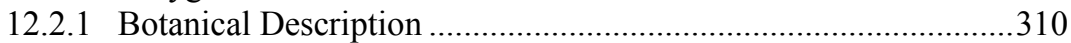

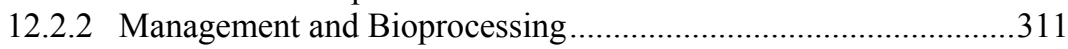

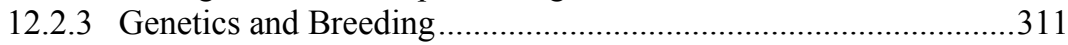

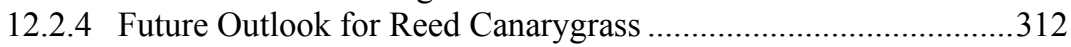

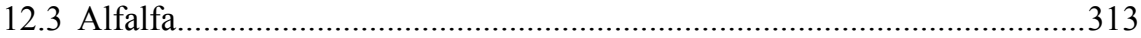

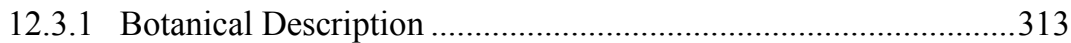

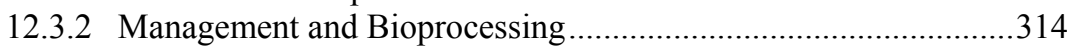

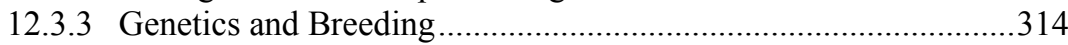

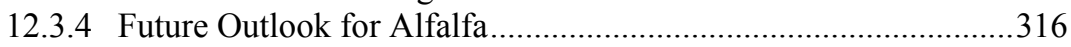

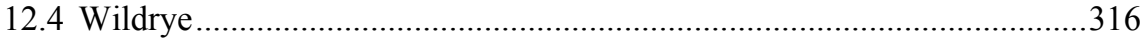

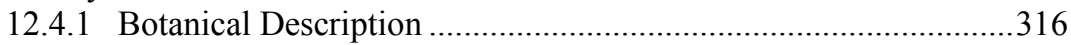

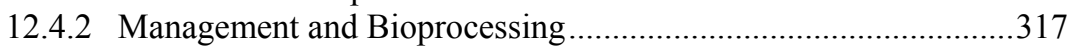

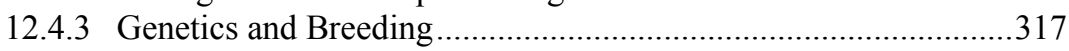

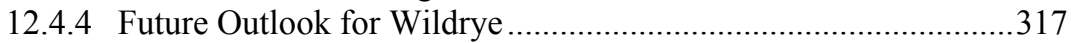

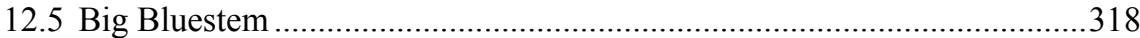

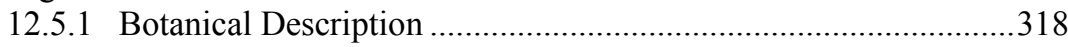

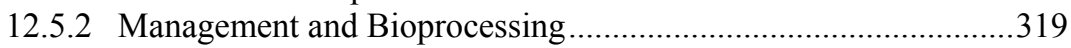

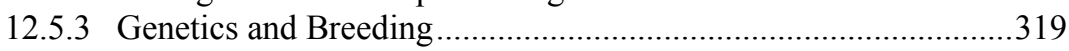

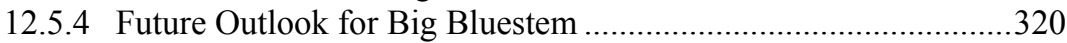

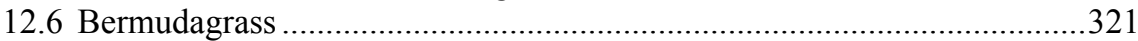

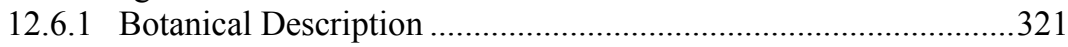

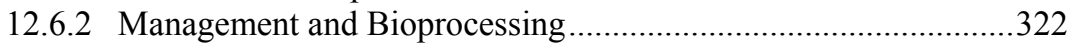

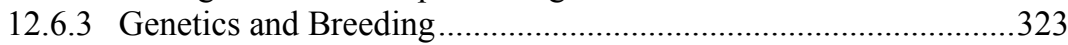

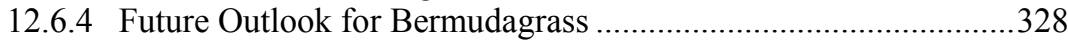

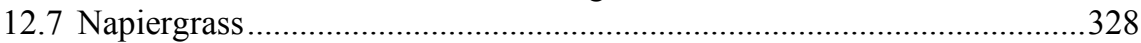

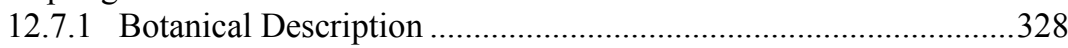

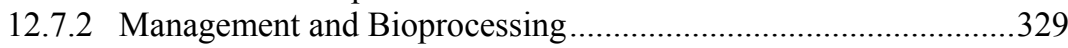




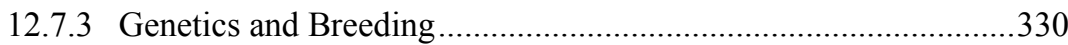

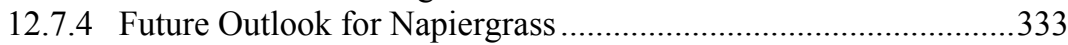

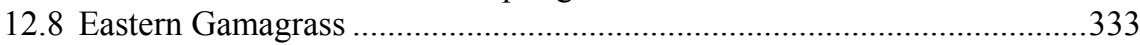

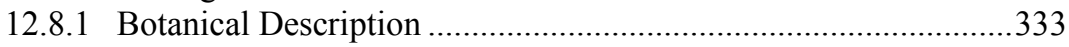

12.8.2 Management and Bioprocessing................................................334

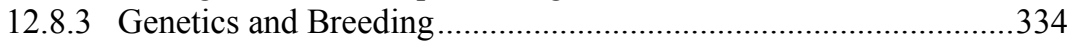

12.8.4 Future Outlook for Eastern Gamagrass ......................................335

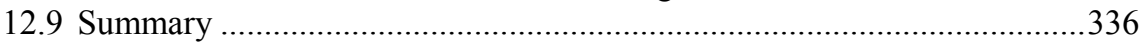

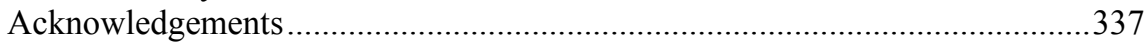

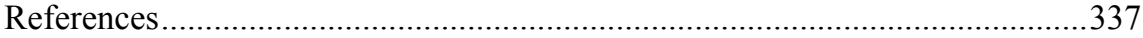

\section{Genetic Improvement of Willow (Salix spp.) as a Dedicated}

Bioenergy Crop ..............................................................................................347

Lawrence B. Smart and Kimberly D. Cameron

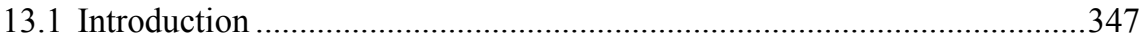

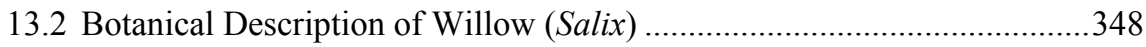

13.2.1 Taxonomy of Species Developed as Bioenergy Crops .................348

13.2.2 Willow Habitat and Growth.........................................................349

13.3 Cultivation, Harvesting, and Processing of Shrub Willow ........................349

13.3.1 Establishing Willow Bioenergy Crop Plantations ........................349

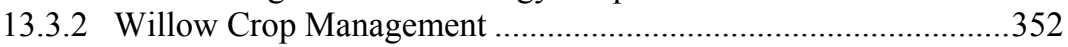

13.3.3 Willow Biomass Harvesting, Transport, and Storage...................355

13.3.4 Processing and Conversion of Willow Biomass to Electricity, Heat, and Transportation Fuels ..............................357

13.4 Breeding and Selection of Improved Shrub Willow

Varieties for Bioenergy ...........................................................................359

13.4.1 Pollination, Hybridization, and Seedling

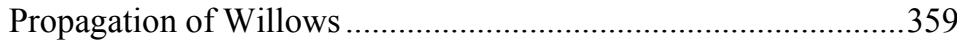

13.4.2 Genetics of Traits Important for Improved Performance of Willow as a Bioenergy Crop .............................362

13.4.3 Current Breeding Efforts for the Development of

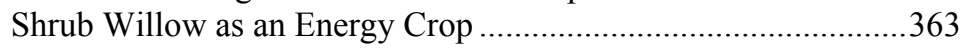

13.4.3.1 Breeding Programs in Sweden........................................363

13.4.3.2 Breeding Programs in the United Kingdom....................364

13.4.3.3 Breeding Programs in North America ............................365

13.4.4 Analysis and Genetic Modification of the Willow Genome.........369

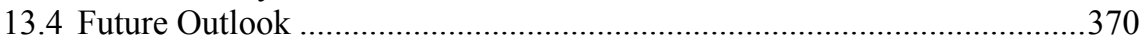

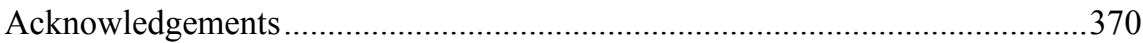

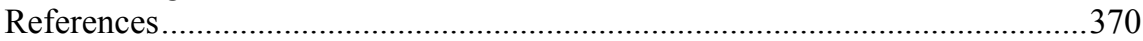

14. Genetic Improvement of Poplar (Populus spp.) as a Bioenergy Crop ..........377 John M. Davis

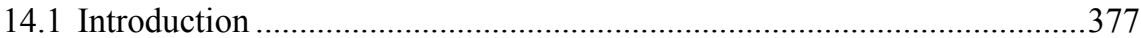

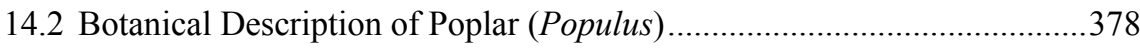

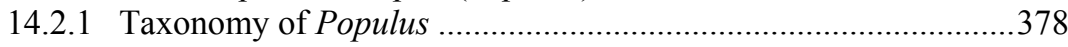

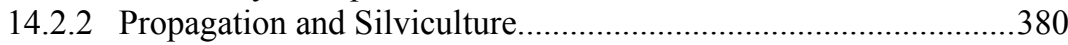


14.2.3 Use of Poplar for Bioenergy …………………...........................383

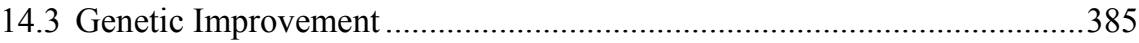

14.3.1 Genetic Parameters for Bioenergy Traits........................................386

14.3.2 Whole-Genome Sequence: The "Parts List" for

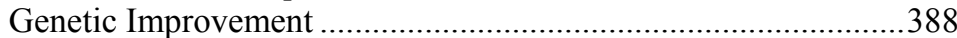

14.3.3 Trait Mapping in Pedigrees and Populations .................................389

14.3.4 Transgenic Alteration of Gene Expression ....................................390

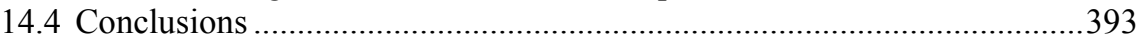

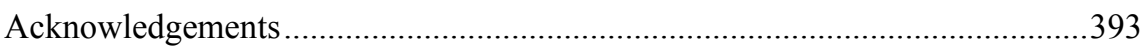

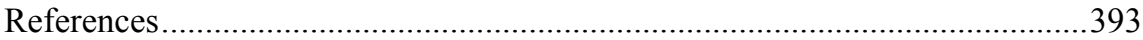

15. Southern Pines: A Resource for Bioenergy .........................................................397

Gary F. Peter

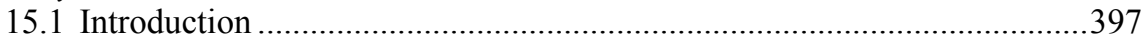

15.2 Botanical Description of Pines: Southern Yellow Pines...............................398

15.2.1 Taxonomy of Species Developed for Plantation Forestry .............398

15.3 Management, Harvesting, Transportation and Bioprocessing

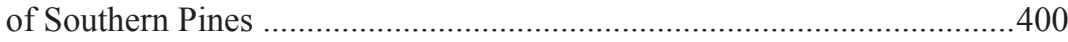

15.3.1 Current Southern Pine Growing Systems for Traditional Forest Products..........................................................400

15.3.2 Southern Pine Harvesting and Transport ........................................403

15.3.3 Silvicultural Research for Short-Rotation Bioenergy Plantings

15.3.4 Traditional Pulp and Paper Bioprocessing Methods, Existing Infrastructure, and Integrated Forest Biorefineries..........404

15.3.5 Stand-Alone Facilities for Converting Southern Pines to

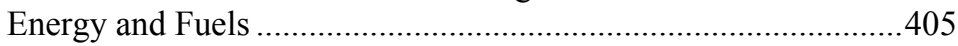

15.4 Life History and Life Cycle of Southern Pines ........................................406

15.5 Genetics and Breeding of Southern Pine ..................................................407

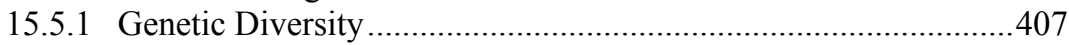

15.5.2 Pollination, Breeding, and Propagation of Southern Pines .............408

15.5.3 Genetic Improvement of Growth, Disease Resistance, and Wood Properties in Loblolly and Slash Pine

15.5.4 Biotechnology in Southern Pines..................................................413

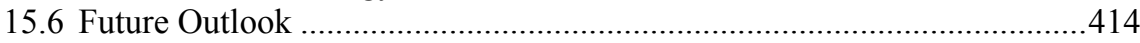

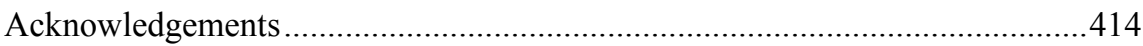

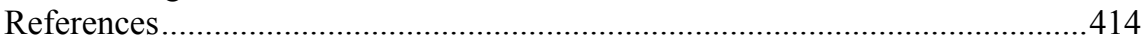

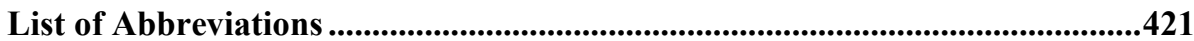

Index 


\section{Contributors}

William F. Anderson

U.S. Department of Agriculture - Agricultural Research Service, Crop Genetics and Breeding Research Unit, 115 Coastal Way, Tifton, GA31793, USA

bill.anderson@ars.usda.gov

Brian S. Baldwin

Department of Plant and Soil Sciences, Box 9555, Mississippi State University, Starkville, MS 39762, USA

bbaldwin@pss.msstate.edu

Rodney J. Bothast

Southern Illinois University-Edwardsville, National Corn-to-Ethanol Research Center, Edwardsville, IL 62025, USA

rbothas@siue.edu

Joe Bouton

Samuel Roberts Noble Foundation, Forage Improvement Division, 2510 Sam Noble Parkway, Ardmore, OK 73401 USA

jhbouton@noble.org

Kimberly D. Cameron

Department of Environmental and Forest Biology, State University of New York College of Environmental Science and Forestry, 246 Illick Hall, 1 Forestry Drive, Syracuse, NY 13210, USA

kdcameron@esf.edu

Michael D. Casler

U.S. Department of Agriculture - Agricultural Research Service, U.S. Dairy Forage Research Center, 1925 Linden Drive West, Madison, WI 53708, USA

michael.casler@ars.usda.gov 
Yu-Chung Chiang

Department of Life Sciences, National Pingtung University of Science and Technology, 1 Shuefu Rd., Neipu, Pingtung 912, Taiwan

yucchiang@hotmail.com

John Clifton-Brown

Plant Breeding and Crop Genetics, Institute of Grassland and Environmental Research, Plas Gogerddan, Aberystwyth, Dyfed SY23 3EB, UK

john.clifton-brown@bbsrc.ac.uk

Robert M. Cobill

U.S. Department of Agriculture - Agricultural Research Service, Sugarcane Research Unit, 5883 USDA Road, Houma, LA, USA

rcobill@srrc.ars.usda.gov

James G. Coors

Department of Agronomy, University of Wisconsin-Madison, 1575 Linden Drive, Madison, WI 53706, USA

jgcoors@wisc.edu

Mark F. Davis

National Bioenergy Center, National Renewable Energy Laboratory, 1617 Cole Boulevard, Golden, CO 80401, USA

Mark_Davis@nrel.gov

John M. Davis

School of Forest Resources \& Conservation, University of Florida, 328 NewinsZiegler Hall, Gainesville, FL 32611, USA

jmdavis@ufl.edu

Natalia de Leon

Department of Agronomy, University of Wisconsin-Madison, 1575 Linden Drive, Madison, WI 53706, USA

ndeleongatti@wisc.edu

Trevor R. Hodkinson

Department of Botany, School of Natural Sciences, University of Dublin, Trinity College, Dublin D2, Ireland

hodkinst@tcd.ie

Tina Jeoh

GeoSynFuels, LLC, 14818 W. 6th Ave \#A1, Golden, CO 80401, USA

tjeoh@msn.com 
Yulin Lu

Department of Agricultural and Biological Engineering, Laboratory of Renewable Resources Engineering, Purdue University, 225 S. University Street, West Lafayette, IN 47907, USA

lu39@purdue.edu

Nathan S. Mosier

Department of Agricultural and Biological Engineering, Laboratory of Renewable Resources Engineering, Purdue University, 225 S. University Street, West Lafayette, IN 47907, USA

mosiern@purdue.edu

Nancy N. Nichols

U.S. Department of Agriculture - Agricultural Research Service, National Center for Agricultural Utilization Research, 1815 N. University Street, Peoria, IL 61604, USA nancy.nichols@ars.usda.gov

Gary F. Peter

School of Forest Resources \& Conservation, University of Florida, 326 NewinsZiegler Hall, Gainesville, FL 32611, USA

gfpeter@ufl.edu

Ana Saballos

Department of Agronomy, Purdue University, 915 W. State Street, West Lafayette, IN 47907, USA

saballoa@ufl.edu

Lawrence B. Smart

Department of Environmental and Forest Biology, State University of New York College of Environmental Science and Forestry, 246 Illick Hall, 1 Forestry Drive, Syracuse, NY 13210, USA

lbsmart@esf.edu

Thomas L. Tew

U.S. Department of Agriculture - Agricultural Research Service, Sugarcane Research Unit, 5883 USDA Road, Houma, LA, USA

ttew@srrc.ars.usda.gov

Ed Wolfrum

National Bioenergy Center, National Renewable Energy Laboratory, 1617 Cole Boulevard, Golden, CO 80401, USA

ed_wolfrum@nrel.gov

Wilfred Vermerris

Genetics Institute and Agronomy department, University of Florida, Cancer \& Genetics Research Complex, 1376 Mowry Road, Gainesville, FL 32610, USA wev@ufl.edu 